\title{
EVOLUTIONARY AND COSMOLOGICAL CORRECTIONS FOR HIGH REDSHIFT GALAXIES
}

\author{
C.S. MÖLLER, U. FRITZE-V. ALVENSLEBEN AND K.J. FRICKE \\ Universitäts-Sternwarte, Göttingen, Germany
}

\section{Abstract}

We calculate the evolution of high redshift galaxies by spectral type with our unified chemically consistent spectrophotometric evolution code. This model takes into account the increasing initial metallicity of successive stellar generations using recently published metallicity dependent stellar evolutionary tracks, color calibrations and spectra (Möller et al. 1997).

We analyse how the consideration of a realistic metallicity distribution affects the evolutionary and cosmological corrections in various bands as a function of redshift. For various spectral types of galaxies and various cosmological parameters we present the color and magnitude evolution as a function of redshift and compare them with observations.

\section{Results}

- For various spectral types E, S0, ..., Sd and different cosmological parameters evolutionary (e-) and cosmological (k-) corrections are calculated in bands UBVRIJHK.

- The comparision between our chemically consistent model and a model with only solar metallicity shows remarkable differences for the spectral types $\mathrm{E}, \mathrm{Sbc}, \mathrm{Sd}$ in various bands.

- We compare observed apparent magnitudes and colors as a function of redshift in optical and NIR bands with our models. The observations are best reproduced by a cosmological model with $\left(\mathrm{H}_{0}, q_{0}\right)=(50,0.5)$.

References

Möller, C.S., Fritze - v. Alvensleben, U., Fricke, K.J.: 1997, A\&.A, 317, 676 